\title{
STUDY OF OCULAR MANIFESTATIONS IN HIV/AIDS CHILDREN IN HIGHLY ACTIVE ANTI RETROVIRAL THERAPY (HAART) ERA FROM BIKANER, NORTHWESTERN INDIA
}

\section{Priya Tanwar *, Gajanand Singh Tanwar, Heena Gahlot, Renu Agrawal; Department of Pediatric Medicine, S.P. Medical College, Bikaner}

\section{Background}

Human Immune deficiency Virus (HIV) has the capability to affect every organ system in the body. Ocular manifestations reflect systemic disease and may be the first sign of disseminated infection. HIV-related eye disease can be classified as retinal HIV microangiopathy, opportunistic infections, neuro-ophthalmic manifestations and unusual malignancies. There is a 52100\% lifetime accumulative risk of HIV patients developing eye problems. Seventy-seven per cent of patients with ocular manifestations of HIV had CD4 counts $<200$ cells $/ \mu \mathrm{L}$. The incidence and type of ophthalmic manifestations of HIV varies worldwide. Highly active antiretroviral therapy (HAART) consists of combination therapy: two nucleoside reverse transcriptase inhibitors and a protease inhibitor which result in substantial and sustained suppression of the HIV replication [HIV RNA viral load]. Highly active antiretroviral therapy has been successful in decreasing the mortality and morbidity of HIV patients. With the patient's immune recovery and increase in CD4 counts, opportunistic infections have a reduced frequency and less aggressive course. All of the available reports concerning ocular involvement in HIV-infected patients in the HAART era relate to adults; no childhood data are available. This prospective study was executed to determine the spectrum of ocular manifestations in HIV positive children in HAART era and to establish their correlation with CD4+Tcells counts.

\section{Method}

This study enrolled 300 HIV positive children from February 2015 to December 2017. These children were evaluated, irrespective of the presence or absence of ocular symptoms and their treatment status. The ocular examination protocol included Visual Acuity [VA] Testing, color vision testing, ocular motility assessment, eye lid and adnexal examination, complete anterior and posterior segment examination. The study was approved by the Ethics Committee of the S.P.Medical College, Bikaner and written informed consent was obtained from the parents or legal guardians of the children.

\section{Results}

Ocular lesions were observed in 50.3\% (151/300) children. Conjuctival microvasculopathy being the commonest finding seen in $9 \%(27 / 300)$ children followed by retinal microvasculopathy $[7.3 \%(22 / 300)]$ and trichomegaly $[6.3 \%$ (19/300)]. Herpes Zoster Ophthalmicus and Cytomegalo virus retinitis were observed in $1.33 \%$ and $1 \%$ children respectively. In this study $78.33 \%(235 / 300)$ children were on HAART. At the time of inclusion in the study, CD4+cell counts ranged from 9 to 1838 cells $/ \mathrm{mm}^{3}$ [mean $\pm \mathrm{SD}=375.6 \pm 249$ cells $/ \mathrm{mm}^{3}$ ]. Low CD4 cell counts and high HIV RNA levels have been associated with ocular involvement and disease progression in HIV-infected children. In the study, 28 children had CD4+cell counts $\leq 100 / \mathrm{mm}^{3}$, in whom $82.14 \%$ had ocular findings, while 71 children had CD4+cell count $\geq 500 / \mathrm{mm}^{3}$ in whom only $35.21 \%$ children had ocular findings. The association between low CD4+cell counts and ocular findings was statistically significant $[p \leq 0.0001]$. We have no HIV RNA data relating to our patients with ocular manifestations because all of them were followed up when viral load was not routinely measured.

\section{Conclusion}

The pattern and severity of ocular manifestations was different than previous reports. Low CD4+cell count and advanced stage of disease are the strong predictors of ocular involvement. This study demonstrates for the first time that the introduction of HAART has had a significant impact on the natural history of ocular manifestations in HIV-infected children, thus suggesting that a reduction in the frequency of ophthalmologic follow-up should be considered for HAARTtreated HIV-infected children with immune reconstitution and no visual symptom. Finally, it is still unknown how long the beneficial effect of HAART on the development of ocular involvement and the risk of progression after previous disease may be sustained. 